ISSN 1409-2441
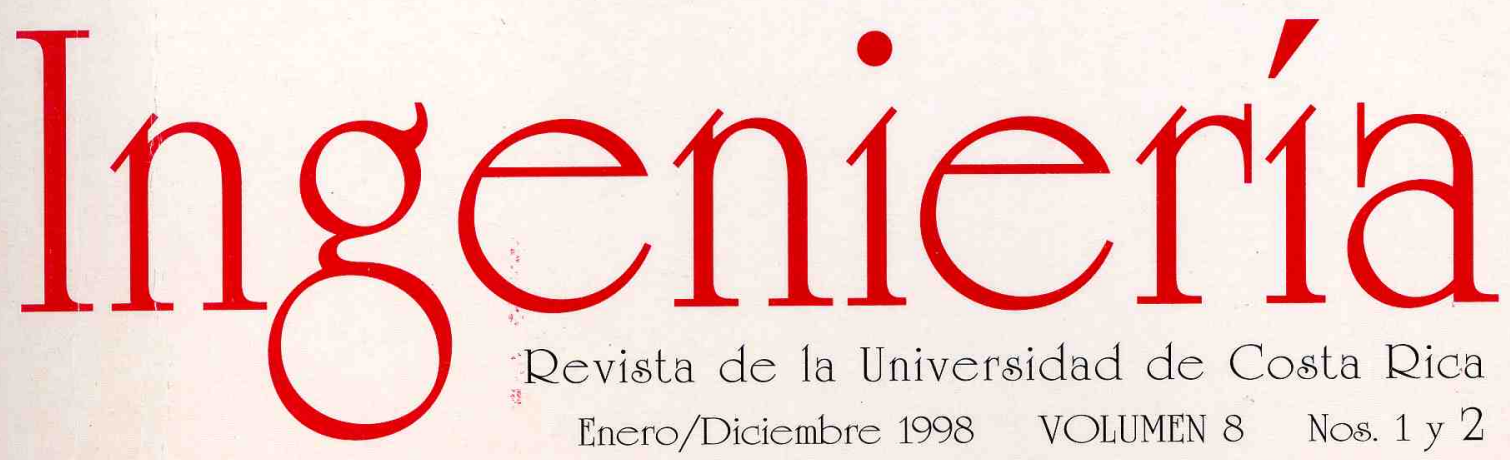

Revista de la Universidad de Costa Rica Enero/Diciembre 1998 VOLUMEN 8 Nos. 1 y 2 


\section{EL DESARROLLO DE UN CATASTRO MUNICIPAL}

;:MartiffChaverrí $R$

\section{RESUMEN}

La municipalidad está, constituida por el conjunto de vecinos residentes en un mismo cantón que promueven y administran sus propios intereses por medio del gobierno municipal (Código Municipal, arto $1^{\circ}$ ). Para ello, el órgano administrativo debe conocer su área geográfica, la propiedad, su uso, nombre y direcciones de sus habitantes. Esto es firnción del catastro. representación gráfica de la propiedad inmobiliaria del cantón en cartas parcelarias \ bancos de datos. con toda la infonnación necesaria, tisica, juridica y personal sobre la propiedad, los contribuyentes, los servicios y vías de comunica<.,'ión. Se hace aquí la propuesta de hacer este trabajo por medio de la empresa privada, para obtener reuttados más rápidamente y se dan las bases técnicas para la re.1lización de este proyecto.

\section{SUMMARY}

"Municipalidad" is a local government for the political division of the province, the "canton". Equivalent to the U.S. county. A new law gave the administration of the land tax to these entities. Most are yet unable to assume this firnction, because they need several instruments, specially a cadastre, to see graphically the parcels of property, locate them by maps and numbers, know the names and adresses of the proprietors. Costa Rica has a National Cadastre, but as it is a complete cadastre: multifmalitary as we call it, advances vaery slowly and we propose here a solution: to contract with a private surveying group, and outline the principal requisites necessary for Ihis kind of work, using the most advanced tedmologies, ;pecially the Land Infonnation System (GIS).

\section{INTRODUCCIÓN}

Creo que las municipalidades son uno de los organismos más democráticos y útiles que podemos tener para el desarrollo, siempre que no se introduzca la politiquería, aunque la experiencia dice que los ciudadanos interesados podrían controlar este aspecto, sin embargo, nos referiremos a eso más adelante.

Parte de la eficiencia de la operación municipal, depende de que esta tenga un buen sistema de catastro. El catastro es la información literal y cartográfica de la propiedad inmobiliaria de una región. Pennite localizar sin lugar a dudas las parcelas de propiedad, entendiéndose por parcela, la unidad de propiedad diferenciada de las colindantes por un lindero poligonal que la rodea, puede ser una cerca de alambre, un muro $\mathrm{u}$ otro material o amojonamiento. La carta parcelaria, muestra las propiedades contiguas, la relación de unas con otras y los accidentes geográficos, como ríos, calles, y otras obras, que circunvalan el grupo. La información literal contiene el nombre de los propietarios, sus datos personales, dirección y relación con el Registro Público de la Propiedad.

La operación de un catastro, aunque sea específica para un país, puede ser útil a nivel transnacional, como fue demostrado en las reuniones convocadas por la Secretaría de Integración Económica Centroamericana (SIECA), durante el desarrollo de nuestro sistema catastral, en década de los 60's, en ese entonces nuestras experiencias mutuas fueron la base del conocimiento que adquirimos sobre esta materia, casi desconocida en el área. Pero más que nada, fue importante la apreciación de las y capacidad, entusiasmo y mística de los colegas de los países vecinos y las amistades de por vida que entonces fOljamos. A nivel técnico, la América Central fue una realidad. Algún día se deberá contar esa historia. 


\section{CLASIFICACIÓN DEL CATASTRO}

Hay muchas clases de catastro:

- El catastro jurídico: debe tener los condiciones para garantizar la existencia del bien, y la demostración de quien es el verdadero propietario.

- El geométrico parcelario: reúne las de buena exactitud dimensionaL con el aspecto jurídico.

- El económico: tiende más a fines de recolección de impuestos que a la exactitud.

- El multifinalitario: por su perfección en todos los aspectos, incluyendo el topográfico, es aplicable a la mayoría de los fines, tanto jurídicos como económicos y de planeamiento de obras.

\section{EL CATASTRO EN COSTA RICA}

El país cuenta con un Registro Nacional, entidad que alberga todos los registros, destacamos el Registro Público de la Propiedad y el Catastro Nacional. Este es del tipo multifinalitario, tiene los requisitos de representación cartográfica de precisión, y los elementos jurídicos para Wla relación con el Registro Público de la Propiedad. Como decía don Alfredo Fernández Iglesias (director del Registro, ya fallecido): "la importancia de esta clase de catastro es que el Registro garantiza la titularidad del bien $y$ el catastro, su existencÍa" .

Aunque se ha realizado un gran trabajo, por los mismos requisitos técnicos y jurídicos que debe cumplir un catastro de esta clase, y su alto costo, etc., no se realiza con la velocidad deseada. Se estima que hay un $30 \%$ del territorio del país cubierto, según información de su director, el ingeniero Jorge Avendaño Machado.

\subsection{CATASTRO MUNICIPAL}

La utilidad del catastro para el municipio es el conocimiento geográfico de su área, las propiedades que en el existen y los datos de los contribuyentes. El poder localizar los propietarios, tener mapas de valores que permitan calcular correctamente el valor asignado a cada propiedad y contener toda la información necesaria para el cobro de las tasas por servicios e impuesto territorial. Además, es deseable contar con los mapas que muestran la topografia y la hidrografia, función del Instituto Geográfico, para proyectar obras y caminos, y muy especialmente, los Planes Reguladores, que definen el tipo de uso más adecuado para un área específica. Es necesario que cada municipalidad cuente con un catastro de su cantón, específicamente para sus fines, que pueden variar de una a otra. El Catastro Nacional ha suministrado a algunas de ellas la información catastral cartográfica, o ha contratado con otras su realización.

Otra opción: Considerando la necesidad de los gobiernos locales de contar con un catastro rápidamente, y no siendo necesarios requisitos estrictos, por ser este un catastro de tipo jiscal, lo puede realizar la municipalidad por contrato con una empresa privada de topografia, trataremos de exponer como se hace, creemos que esto es de aplicación general.

\subsubsection{FACTORES PARA TOMAR EN CUENTA}

Condiciones físicas y económicas: Hay Costa Rica 51000 kilómetros cuadrados, dividido el país políticamente en 7 provincias y 80 cantones; el gobierno local del cantón es la municipalidad. Encontramos que hay grandes diferencias de uno a otro cantón, tanto en extensión como en la constitución de la población, la clase de desarrollo: urbano, rural: agropecuario o agrícola.

Fidelidad del trabajo: este puede tener diferentes grados de exactitud geométrica, según los medios cartográficos y económicos 
de que se disponga, debe definirse también la precisión jurídica; lo que interesa, para este tipo de catastro, antes de la demostración y prueba concluyente de quien es el verdadero propietario, y defensa de terceros, proceso engorroso, necesario en el catastro jurídico, es quien va a pagar los impuestos, donde vive, cuanto vale su terreno, uso del mismo, etc. Claro que es prudente la mayor exactitud en este sentido, tanto para la relación con el Registro Público, como para cualquier reclamo de la Municipalidad.

o Aspectos legales: Como lo hemos dicho, el catastro localiza sin lugar a dudas la parcela contribuyente, obsérvese que difgo: parcela contribuyente porque se individualiza la parcela, por cuanto se refiere a un impuesto in rem (real), que es el caso del territorial (la parcela responde por el impuesto sobre la misma). Paralelamente a la información cartográfica parcelaria, debe existir la infornlación literal de datos de varias clases, cuyas fuentes deben ser, primero los planos "catastrados" (llamamos así a los planos de agrimensura inscritos en Catastro), la escritura o folio real correspondiente, dirección del propietario (o su representante), y segundo, la misma municipalidad, en su tarjetero para el cobro de servicios. Es necesaria una relación catastro - banco de datos de la administración municipal (estado de los cobros por servicios, patentes, territorial, etc.).

o Organización del municipio: Para lo que interesa, esta es la corriente de estos organismos: un Concejo, compuesto por regidores de elección popular; un alcalde. también de elección popular; un sistema administrativo y otras dependencias; entre las que se cuenta ingeniería, de la que por lo general, depende el catastro, necesario para contar con el archivo de planos y la información registral usada para otorgar los permisos de construcción, urbanizaciones y plan regulador (para la zonificación del cantón). A cargo de ingeniería están los departamentos de obras, mantenimiento, servicios y un grupo de inspectores para la vigilancia de las obras, como construcciones, urbanizaciones, etc. La cantidad de personal y dependencias depende desde luego, del tamaño, población y desarrollo del cantón.

Los municipios rurales, no tienen mucho personal ni equipo de cómputo, ni departamentos especializados. Al no contar con catastro, llevan la infonnación de contribuyentes en un simple fichero de tarjetas con los datos necesarios para el cobro de los servicios, es lo que se llama un «amillaramiento». Una entidad que ha trabajado en el desarrollo de los municipios es el Instituto de Fomento y Asesoría Municipal (IFAM), a la que deben muchas municipalidades, un sistema de catastro elemental, consistente en croquis de las manzanas urbanas, se mide el frente de cada propiedad con cinta métrica y se investiga y anota el propietario. Los frentes de propiedad son la base para el cobro de las tasas por servicios de agua, luz, basura, limpieza, entre otros.

Declaración de bienes: En la nueva ley de impuesto a los bienes inmuebles, (ley 7509 de junio de 1995), se procedió a realizar un censo con la declaración volwHaria de los propietarios, y ha dado un resultado bastante bueno, con la combinación de un avalúo masivo del cantón, y la comparación de la información dada por el propietario sobre el valor de su propiedad con el que se obtiene del mapa de valores y adicionalmente se obtienen los planos catastrados, que ya tenían la mayoría de los propietarios. Sin embargo, la aplicación de esta ley coincidió con la agitación política de las elecciones de 1998, presidenciales y de diputados, lo que fue aprovechado por la Asamblea Legislativa para reducir inconsultamente la tasa del impuesto a niveles tan bajos, que promueve la especulación con tierras, ya que es posible tener grandes cantidades de tierras sin explotar, en espera del aumento constante de 
su valor, por el desarrollo, por cuanto lo que se paga en el impuesto es mínimo, obteniendo así el propietario un aumento del valor al que no ha contribuido (incremento no ganado). Un impuesto adecuado (basado en estudios del valor y efecto del mismo en el desarrollo), a la tierra desnuda (sin tener en cuenta las mejoras en lo rural), promueve su uso y baja el costo de la tierra para quienes la necesiten para su trabajo. Tal cosa la propuso uno de los mejores estudios realizados en este país "Impuestos inmobiliarios rurales de Costa Rica, un anális legal y económico" , por la Facultad de Derecho de la Universidad de Costa Rica, y otros.

\section{METODOS DE LEVANTAMIENTO}

Dependen mucho de las posibilidades y valor económico del Municipio, por lo general, son las áreas urbanas las que reciben atención prioritaria. Como decíamos, el Catastro Nacional, está contratando con algunos municipios la realización del catastro de su cantón. Tiene la ventaja de que se hace un trabajo de primera calidad, lo que desde luego es más costoso. No hablaremos de los métodos clásicos, de sobra tratados más ampliamente en libros al respecto, ni del catastro fiscal sobre ampliaciones de fotografia aérea, porque en un país montañoso, como el nuestro, se producen distorsiones perspectivas muy grandes que no permitirían su mantenimiento.

El plano "catastrado": Es prescripción legal que toda operación con la propiedad, hipoteca, venta, herencia, donación, litigio, etc., sea sustentada por un plano de agrimensura que describa en su forma dimensiones y localización, la parcela de la propiedad afectada. Estos planos, están referidos a los mapas topográficos del Instituto Geográfico, y tienen la distancia medida de uno de sus vértices a un punto específico del mapa. Se presentan al Catastro, que los revisa, en cuanto a localización y datos de escritura, se sellan, se archivan y se dan copias selladas al agrimensor, se requieren con frecuencia visas no solo de catastro, sino también de la municipalidad del cantón, si es para obtener un permiso de construcción, o una patente, o por el Instituto Nacional de Vivienda y Urbanismo (INVU), para un proyecto de urbanización, subdivisión por herencia, condominio, u otro motivo.

El movimiento en catastro para estos efectos es grande y la actividad mantiene ocupados a más de mil agrimensores. Se estima que hay más de dos millones de planos de la propiedad inmobiliaria algunas están "catastradas" varias veces, y por tanto, es un precioso recurso para hacer las cartas parcelarias para el catastro municipal, haciendo el montaje como se explica más adelante. Recuerdo haber leído que en Uruguay se emplearon estos planos para hacer el catastro del país, de modo que no es nada nuevo.

Contrato: Para hacer el catastro el municipio debe específicar en la solicitud pública de servicios, la competencia y experiencia de la empresa oferente. Esta competencia debe radicar,. no solo en la capacidad académica de los oferentes, sino también en el conocimiento del catastro y el municipio, sus necesidades y posibilidades, como en la posesión del equipo topográfico adecuado: teodolitos y computadoras electrónicas, GPS, el sistema de localización por satélites, es recomendable y aún más, un sistema de información geográfico (SIG).

El contratista debe realizar un díagnóstico del municipio, como parte básica de su proyecto, en cuanto a:

1. Existencia y operación del departamento de ingeniería (¿archiva los planos dt agrimensura y los de urbanizaciones?).

2. Existencia y operación, si existe, de un catastro municipal (por lo general existen, aunque no completos). ¿Tiene sección de evaluación y mapa de valores?

3. Relación del catastro municipal con el departamento administrativo de cobros para la actualización de estos: a)lista de 
contribuyentes y direcciones, b) cobro de tasas por servicios.

4. Sistema contable y de cómputo, si existe.

5. Personal (Ingeniería y catastro), calificación del mismo. ¿Se puede usar personal contratado localmente para el trabajo?

6. Espacio fisico, equipo y mobiliario para el catastro a desarrollar.

7. Todo otro aspecto relacionado con el proyecto, como: mapas topográficos existentes, mapas de estadística y censos con los linderos cantonales, etc.

Basado en el análisis anterior, hacer el presupuesto, considerando los aspectos técnicos, y lo que se quiere hacer. Desarrollar y presentar un esquema y organización del trabajo.

\section{EL LEVANTAMIENTO TOPOGRÁ- FICO}

No se entra en los detalles técnicos, que toda empresa contratista de esta clase de trabajos conocerá, además depende de la localización del municipio, por cuanto no es lo mismo hacerlo para un municipio rural y de pocos medios, que para uno del área central y desarrollada del país, que necesitan algo más exacto. Tendrá algunas diferencias en cuanto al levantamiento urbano y el rural. Pero ambos deberán estar enlazados a la red de control geodésico del país y al sistema de proyección cartográfica que se detennine. El enlace puede hacerse por medios clásicos, como la triangulación o una poligonal a puntos amojonados de la triangulación del país, o por medios más modernos, como el GPS (GEODETIC POSITION SYSTEM), por la observación de satélites y permite localizarse en fonna más rápida y segura que por los otros medios de la geodesia. Las autoridades (Instituto Geográfico, Catastro Nacional) deberán definir los enlaces y demás parámetros pertinentes. Los puntos de control, en el caso del levantamiento urbano, se localizaran en las esquinas extremas de la ciudad o población, dependiendo de su ex1ensión y forma. Deberán amojonarse de modo que constituyan puntos de referencia lo más permanente posible, el amojonamiento debe ser sistemático y también requiere de una campaña para incentivar a los propietarios a cuidar los mojones, de la red original de control del Instituto Geográfico Nacional, están quedando pocos hitos de referencia, lo que aumenta los problemas y costos del agrimensor y topógrafo.

5.1 En el levantamiento urbano se correrán poligonales que permitan el levantamiento geométrico de todas las manzanas de la población. Si las manzanas son regulares el levantamiento será más simple, usando una cuadrícula de levantamiento que podría ser en líneas paralelas cada dos manzanas y desde los puntos de intersección marcar con puntos marcados con clavos o pines de bronce. Desde estos puntos de referencia en las esquinas de las manzanas, se localizará la línea de propiedad, sobre ella con cintas, un equipo de trabajo consistente de dos cadeneros y uno o dos enwneradores, medirán los frentes de cada propiedad e interrogarán a quienes viven en ellas para obtener los datos sobre los propietarios. Esto requiere una fuerte campaña de divulgación, con el fin de que el trabajo de los enumeradores no encuentre muchos obstáculos.

\subsection{Formulario de investigación: Según lo} anterior además del levantamiento topográfico, se requiere una investigación de datos del propietario y la propiedad, en su diseño debe contarse con las especificaciones y necesidades de la municipalidad.

\subsection{Dibuio $y$ Montaje de Planos: La} generalidad de los levantamientos topográficos en la actualidad se hacen en un procesador con un programa $\mathrm{CAD}$, de los que en el mercado encontramos varios sistemas, algunos muy sofisticados y costosos, la selección dependerá del presupuesto asignado y necesidades del municipio. 
De los levantamientos de manzanas obtenemos los mapas del perímetro de las mismas (VER DIBUJO), con la indicación de los frentes a la calle de las parcelas, que deberán ser en escala grande, usualmente en 1:1000- sobre estos, se montan los planos de agrimensura, obtenidos del catastro o del propio archivo de ingeniería municipal, para fonnar el mapa parcelario. $\mathrm{La}$ información se obtiene de la digitación de los planos individuales o del cálculo de las coordenadas, que luego pueden transformarse, para adaptarlas a las del levantamiento, aunque es más rápido y suficiente el montarlas y girarlas hasta que coincidan unas con otras, visualmente. Es seguro que de primer momento no habrá 1Ula coincidencia muy buena en el mosaico, debido a que esto requiere que cada agrimensor mida sobre hitos de marca de linderos, acordados por los propietarios colindantes, y aunque esto está en la ley, solo en caso de litigio se hace, además, los levantamientos deberían estar enlazados todos a un sistema uniforme de coordenadas, derivadas tal vez, de puntos de referencia amojonados en las esquinas de las manzanas.

Para los fines del uso municipal, basta el procedimiento descrito de acomodo visual de las parcelas, salvo en casos de gran discrepancia, que deben completarse con una revisión de campo, a cargo del propietario o como responsabilidad del agrimensor, si es por defecto de este.

5.4 Levantamiento rural: En prinCipIO, es semejante a lo expuesto para el levantamiento urbano, se levantan bloques entre calles, o calles y ríos (se les llama "islas". Estos levantamientos pueden hacerse tan rápidamente con los instTunlentos modernos, que en áreas de mediana densidad rural y con buenas carreteras, es lo más recomendado.

5.5 Fotogrametría: En las áreas rurales, de grandes propiedades, de terreno agreste y pocos caminos, es casi el sistema más económico. Se requiere amplia experiencia y conocimientos para la planificación del mismo. También se ha usado con éxito en levantamientos urbanos, y todas nuestras ciudades del área metropolitana de Costa Rica, han sido levantadas por este método.

Se requiere un plan de vuelo para obtener fotografia aérea vertical con cámaras métricas de precisión, según lo determinen las especificaciones- En el área urbana las fotografias pueden ser de escala aproximada de 1:5000 a 1:10000, para restitución a 1:1000. En las zonas rurales puede trabajarse, siendola densidad predial media de 30 a 100 hectáreas 0 algo más, con fotografia en escala de 1:40 000., para restitución en escalas de 1:5000 a 1:10000.

La identificación y localización de puntos de control para la restitución de la fotografia aérea, se obtiene más fácil y rápidamente por el método GPS, de localización por satélites.

Es recomendable por la mayor rapidez y seguridad de trabajo en el campo, la transformación de las fotografias (vistas perspectivas), en ortofotos, vistas ortogonales, como los mapas.

\subsection{La delineación y obtención de datos:}

Esto se hace por enumeradores, con ampliaciones de las ortofotos a las escalas adecuadas, los que deberán investigar los propietarios, seguir y marcar en la fotografia los linderos de las propiedades y obtener toda la información pertinente. Para esto, la experiencia, a veces amarga y molesta, nos dice que es necesaria una intensa campaIlade divulgación de los fines del trabajo que se realiza y que son los datos que necesita el propietario tener a mano.

\section{SISTEMAS DE INFORMACiÓN GEOGRÁFICA (GIS)}

Todos los que han tenido oportunidad de utilizar y trabajar con los sistemas de información geográficos, están de acuerdo de su utilidad en las municipalidades. "Es un Sistema de Información diseñado para trabajar con datos espacialmente referenciados. En otras palabras, un Sistema de Información 
Geográfica es tanto un sistema administrador de base de datos con capacidad para referenciar espacialmente los datos, como un conjunto de operaciones para trabajar con ellos.

Aunque los SIG por si mismos son una poderosa herramienta con la que se pueden hacer gráficos o mapas, su potencial está en la capacidad de poder identificar dentro de estos mapas, de lila fonna fácil toda la infonnación que pueda estar ligada a su ubicación geográfica, en el se puede mostrar y analizar la misma y los datos literales correspondientes. Pueden obtenerse varios resultados como:

1. Localización de los propietarios y servicios relativos.

2. Detectar áreas para nuevos desarrollos y zonas de conservación.

3. Localizar acuíferos y cuencas hidrográficas, etc.

4. Confeccionar mapas con información de diferentes fuentes y realizar análisis para diferentes fines.

5. Tomar decisiones sobre donde y cuando ubicar los recursos.

6. Actualizar automáticamente todos esos mapas y planos cuando ocurran cambios en la información.

7. Se pueden ligar las características y elementos asociadas a ellas, tales como locales comerciales, instituciones, zonas agrícolas, ríos, calles, escuelas, hospitales, fallas geológicas, etc."

Creo que la importancia del SIG en un municipio es su agilidad para dar servicio a todos los departamentos del organismo, en "irtud de poder ser estructurado cartográficamente en capas, las que pueden superponerse según las necesidades específicas de estos mismos. Se señala:
- Capa de la cuadrícula de la proyección escogida.

- Capa de los puntos de la red de control geodésica (horizontal y vertical) del área.

- Capa de la hidrografia.

- Topografía, curvas de nivel.

- Obras de arte (obra del hombre), carreteras, puentes, calles, casas, edificios, obras hidráulicas, muelles, etc.

- La capa parcelaria, con las parcelas de propiedad numeradas y ligadas a la información pertinente.

- Mapas del Plan Regulador.

- Mapa de valores.

Más, acueductos, alcantarillado, y todo lo necesario para el gobierno local.

Como todo esto "iene acompañado de un banco de datos, tan completo como sea necesario, veremos a la Administración relacionando el mapa parcelario con el cobro de tasas, direcciones, etc. A Patentes con el uso y permisos de operación de negocios, oficinas, bares, etc. $Y$ sobre todo a Ingeniería administrando por medio de los mapas del plan regulador, el cumplimiento del mismo y proyectando las obras necesarias con los mapas topográficos $\mathrm{y}$ de control.

6.1 Actualización: Es una de las operaciones más importantes y la cual deberá quedar totalmente establecida y funcionando desde el primer día. Debe desarrollarse desde dos aspectos diferentes:

a. por medio de una relación con el Registro de la Propiedad y el Catastro Nacional, que infonnen diariamente de los movimientos de las propiedades, información que puede darse desde el sistema de cómputo del Registro.

b. desde la Ingeniería municipal, por medio de los permisos de construcción

6.2 El mapa de valores: Es desde luego parte muy importante del catastro municipal, le permite al funcionario encargado, la 
evaluación sistemática y uniforme de los predios, es un tema que no desarrollamos, porque en Costa Rica ha sido función de la Dirección General de Tributación Directa, la que con motivo de la declaración voluntaria de bienes inmuebles, en 1996, envió peritos valuadores a todas las municipalidades, y prepararon el mapa de cada cantón y entrenaron el personal en su uso y el cálculo de los valores de las construcciones.

Creemos y estamos de acuerdo con muchos economistas que así lo han manifestado, que en lo urbano debería eXlstlr una tarifa diferencial, con una tasa menor para las construcciones y mayor para la tierra, puesel aumento constante del valor de esta, la renta de la tierra, es lo que propiamente corresponde al Estado. En lo rural, la renta de la tierra debe ser el único impuesto, pues como lo manifiesta el Dr. Kalninus en su estudio sobre la imposición territorial en Costa Rica, cobrarlas mejoras y la producción agrícola, es castigaral que trabaja.

\section{CONCLUSIONES}

No se ha presentado un tratamiento exhaustivo, de una materia sobre la que se han escrito muchas obras, queremos presentar a las personas interesadas en el desarrollo municipal y regional, la posibilidad de que los gobiernos locales puedan realizar un trabajo de tanta importancia y utilidad como es el catastro, por medio de la empresa privada y el empleo de personal local.

\section{BIBLIOGRAFÍA}

1. Aguilar V., D. Introducción a los Sistemas d Información Geográfica, 1998

2. Álvarez F., E. Aspectos que debe conocer el profesional encargado del Departamento de Catastro en una Municipalidad, $\quad 3^{3}$ Conferencia de Topografia, Colegio de Ingeniero Topógrafos de Costa Rica. 1998.

3. Chinchilla V., E. IFAM Y El Impuesto Territorial, Instituto de Fomento y Asesoría Municipal, 1985., Código Municipal, Publicado en la Gaceta N" 94 del 18 de mayo de 1998.

4. Jung, G. Los Avances Obtenidos en Materia Catastral,fundamentalmente en la evaluación del Catastro Multifinalitario, 1998.

\section{Martín Chaverri Roig \\ Survey Engineer \\ P.O.BOX 1939 JODO}

Email: macharo@terraba.fing.ucr.ac.cr

San José, Costa Rica

Phone: (506) 224-32-92 